\section{Safety, pharmacokinetics and efficacy of artemisinins in pregnancy}

\author{
Veronica Ades \\ University of California, San Francisco, \\ CA, USA
}

Abstract

Malaria in pregnancy can lead to serious maternal and fetal morbidity and mortality. Access to the most effective antimalarials in pregnancy is essential. Resistance to current therapies is high for all antimalarial therapies except artemisinins. Artemisinin-based combination therapy is current the first line of malaria treatment recommended by the WHO for children, adults and pregnant women in second or third trimester. Due to potential embryotoxicity of artemisinins identified in animal studies, artemisinins are not considered safe for use in first trimester of pregnancy. Artemisinins are more rapidly metabolized in pregnant women, but it is not clear whether this reduces efficacy. Most studies show very high cure rates for pregnant women. Areas for further research include the safety profile in first trimester of pregnancy, the effect of HIV infection on artemisinin use in pregnancy, the relationship between the pharmacokinetic profile and efficacy, the effect of newly emerging artemisinin resistance on treatment in pregnancy and the use of artemisinin-based combination therapy for intermittent preventive treatment in pregnancy.

\section{Introduction}

Malaria in pregnancy has been associated with poor pregnancy outcomes, including intrauterine growth restriction, low birth weight, spontaneous abortion, neonatal death, preeclampsia, maternal anemia, and maternal death. ${ }^{1-3}$ Diagnosis of malaria in pregnancy is compromised by low parasitemia burdens associated with sequelae. Specific malaria parasites express variant surface antigens (VSA) that mediate adherence to placental chondroitin sulfate A (CSA), leading to placental sequestration of parasites, continual placental damage, and inability to clear parasites through normal immunity. ${ }^{4}$

Primigravid and secundigravid women are most susceptible to the maternal and fetal effects of malaria in pregnancy, especially in areas with stable transmission. ${ }^{5}$ Women with HIV are susceptible regardless of gravidity. ${ }^{6}$
Malaria infection in pregnancy may not result in clinical symptoms; however, when symptomatic malaria does occur in pregnant women, the risk of severe clinical sequelae is higher than in non-pregnant adults., ${ }^{2,7}$ Therefore, it is particularly important to have effective means of treating symptomatic malaria in pregnancy.

A variety of medications have been established as safe and effective in pregnancy, including chloroquine, quinine, sulfadoxinepyrimethamine, mefloquine, chlorproguanil, dapsone and amodiaquine. ${ }^{5}$ However, increasing resistance to these agents has led to a need for more effective malaria treatments for both children and adults. ${ }^{8}$

Artemisinin, an extract of the Artemisia plant found in China known as qinghaosu, was identified and tested in China in the 1970s for use by the North Vietnamese army during the Vietnam War. The Artemisia plant had long been used in China to treat fever, and was often consumed whole by indigenous people when ill with malaria. In the 1980 s, the Chinese army found that combining artemisinins with other antimalarials led a reduction in recrudescence after treatment. ${ }^{9}$

Artemisinins were not made available to the Western world until 1999, and were added to the WHO List of Essential Medicines in 2001. ${ }^{9}$ Artemisinin derivatives include artesunate, artemether, and dihydroartemisinin. Resistance to artemisinin-derived compounds remains at a minimum, having only been detected in Asia along the Thai-Cambodia border. $^{10}$

Artemisinins are both highly potent and rapidly eliminate parasitemia. Artemisinins are usually given in combination with another antimalarial agent with a longer half-life to eliminate remaining parasites. ${ }^{10}$ Artemisininbased combination therapies are currently the first-line recommended treatment for malaria in pregnancy in second and third trimesters. The WHO-recommended first-line treatment for malaria in first trimester of pregnancy is quinine plus clindamycin. ${ }^{11}$ Data are limited on the effects of artemisinin-derived compounds in pregnancy. They are considered to be safe in second and third trimesters, but still requires further evaluation to determine if they are is safe in first trimester. Data from animal models suggest that they are embryotoxic in rats and rabbits through inhibition of hematopoiesis and angiogenesis, but it is unclear if this effect is present in human embryos. Various studies of artemisinins given inadvertently in first trimester have conflicting results regarding association with poor pregnancy outcome. This review will summarize the current evidence regarding the safety, pharmacokinetics, dosing and efficacy of artemisinins in pregnancy.
Correspondence: Veronica Ades, 220 N. Zapata Hwy, Laredo, TX, 78043, USA.

E-mail:va77@mac.com

Key words: malaria, pregnancy, artemisinin.

Conflict of interest: the authors report no conflicts of interest.

Received for publication: 20 March 2011.

Revision received: 14 May 2011.

Accepted for publication: 18 May 2011.

This work is licensed under a Creative Commons Attribution NonCommercial 3.0 License (CC BYNC 3.0).

(C)Copyright V. Ades., 2011

Licensee PAGEPress, Italy

Infectious Disease Reports 2011; 3:e8

doi:10.4081/idr.2011.e8

\section{Choice of agent}

\section{Artemether}

Artemether was the first artemisinin derivative to be evaluated on a large scale. ${ }^{12}$ It is most commonly administered in an oral co-formulation of artemether-lumefantrine and is a highly effective treatment for children and adults with uncomplicated malaria. ${ }^{11}$ Because it is a fat-soluble compound, artemether is not ideal for intramuscular or intravenous administration, limiting its utility for severe malaria, which requires parenteral treatment. ${ }^{12}$

The only pharmacokinetic study on artemether in pregnancy shows reduced plasma concentrations of artemether in pregnancy. ${ }^{13}$ Five prospective studies have been published on the clinical effectiveness of artemether in pregnancy. ${ }^{14-18}$ Four of the studies show good clinical effectiveness, while one study found a high rate of failure for both primary and recrudescent infections (Table 1). Three animal studies evaluated the possible toxicities of artemether in pregnancy. Two of them showed dose-dependent embryotoxicity, and the third further investigated various reproductive effects of artemisinins on ovulating and pregnant rats. ${ }^{19-21}$ One human study regarding safety had conflicting data regarding first-trimester exposure, but found no adverse effects of second- or third-trimester exposure. ${ }^{22}$ These studies are discussed more indepth in this paper.

\section{Artesunate}

Artesunate, a water-soluble compound, is more stable for parenteral administration, and can be given in severe malaria. It is available as intravenous monotherapy, or in oral co-formulations as artesunate-mefloquine or artesunate-amodiaquine. Four animal studies involving artesunate show similar patterns of anomalies and embryotoxicity seen with all artemisinin derivatives. ${ }^{23-26}$ 


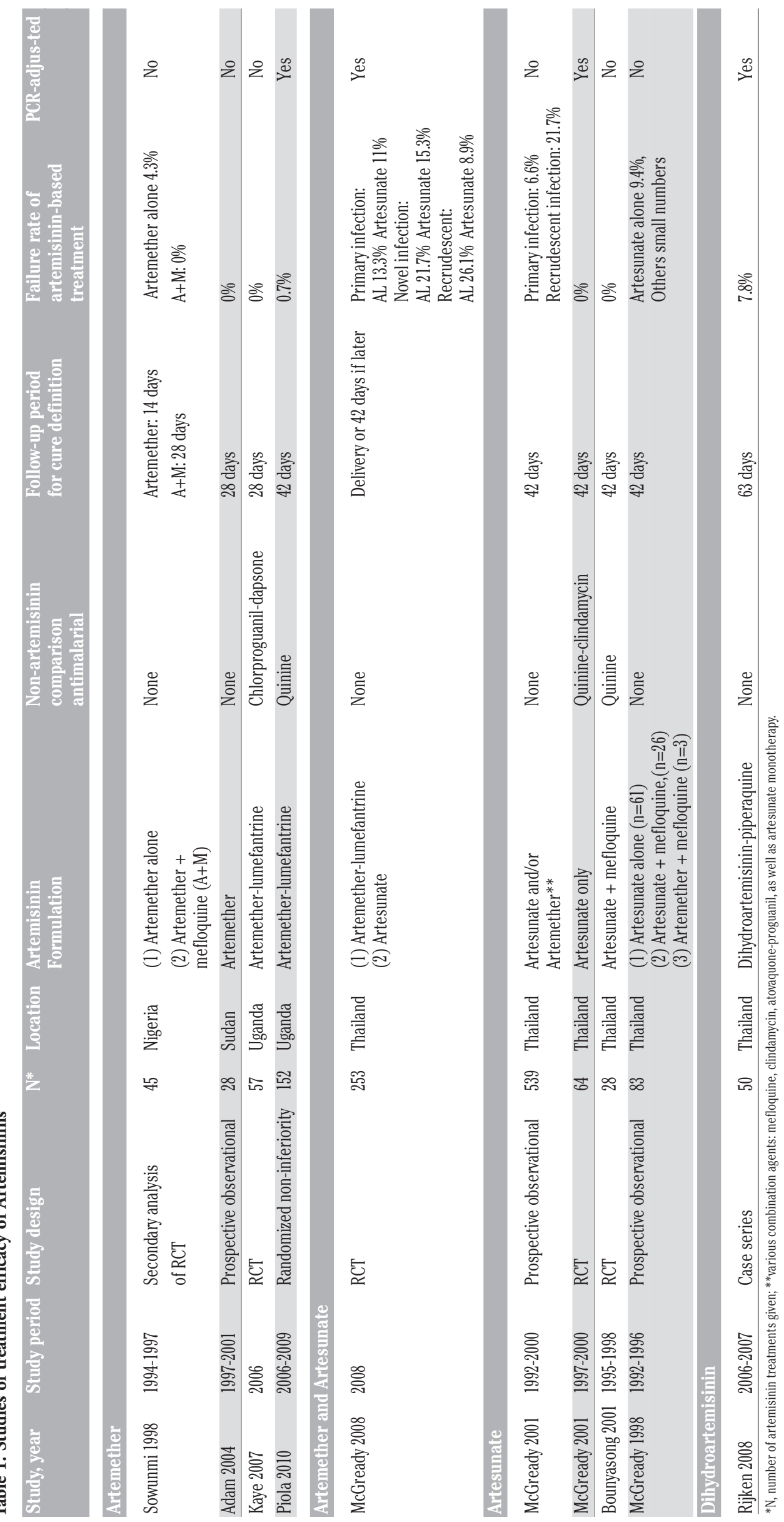


A human safety study retrospectively evaluated a subcohort of pregnant women who received artesunate $v s$ sulfadoxinepyrimethamine as part of a community mass drug administration study, including 77 women who were exposed during first trimester. The study found no difference in pregnancy outcomes between those given artesunate and those given sulfadoxinepyrimethamine, although the study was not designed to adequately detect first-trimester spontaneous abortions. ${ }^{27}$ Two pharmacokinetic studies of artesunate showed lower plasma concentrations of the artemisinin active metabolite in pregnancy, but both studies also had high rates of effectiveness, and therefore it is unclear whether this pharmacokinetic difference is manifested clinically. ${ }^{28,29}$

Among the seven treatment efficacy studies on artesunate, most showed efficacy rates of $100 \%$ when artesunate was combined with another antimalarial agent, and those that used artesunate as a monotherapy had variable failure rates . One study showed higher recrudescence (21.7\%) for established recrudescent malaria. ${ }^{27,30-34}$ A 2008 study showed that artesunate may be a superior to artemether-lumefantrine in recrudescent malaria infection. ${ }^{16}$

\section{Dihydroartemisinin}

Dihydroartemisinin is available in an oral co-formulation with piperaquine. Dihydroartemisinin is also the major active metabolite of several artemisinin derivatives. ${ }^{35}$ Three animal studies have been published on dihydroartemisinins in pregnancy, suggesting that the mechanism of teratogenicity lies in its anti-angiogenic activity during yolk sac hemotopoeisis. ${ }^{23,35,36}$ To date, there is only one study of treatment efficacy of dihydroartemisinin in pregnancy, and that study shows a treatment efficacy of $92.2 \%{ }^{37}$

\section{Safety}

\section{Animal studies}

Studies in rats and rabbits have demonstrated the embryolethality and teratogenicity of artemisinin derivatives. Three studies from China in the 1980s and 1990s showed possible embryotoxic or teratogenic effects of artemisinin derivatives. A study by Chen et al. found that artemether caused embryo resorbtion in mice when given in high doses (10.7 and $21.4 \mathrm{mg} / \mathrm{kg}$ ) but no adverse effects were seen at low doses $(0.7$ and $5.4 \mathrm{mg} / \mathrm{kg}){ }^{19}$ Another study by Xu et al. in 1996 found that the ED50 dose of artemether cause embryo absorption in mice and rabbits, and induced abortion in guinea pigs and hamsters. ${ }^{23}$

Two studies evaluate the dose-response effect of artemisinins on embryolethality. Longo et al. showed that rat embryos exposed to dihydroartemisinin, the major metabolite of artemisinin derivatives, had a dose-dependent interference with hematopoiesis and angiogenesis by DHA. ${ }^{36}$ Li et al. showed that the embryolethality of artesunate administered intramuscularly and intravenously corresponds to pharmacokinetic findings of high peak concentrations. ${ }^{26}$

In 2004, Clark et al showed that artemisinins caused not only embryolethality, but also congenital anomalies in rats. Groups of 24 pregnant rats were given chlorproguanildapsone-artesunate versus artesunate alone and were compared with groups of control rats. Both the artesunate combination and the artesunate monotherapy caused postimplantation fetal loss, as well as cardiovascular and skeletal anomalies. The experiment was repeated with rabbits, and found the same outcomes. The reported adverse outcomes were most pronounced in doses substantially higher than human doses, and no maternal toxicity was seen at any dose. ${ }^{24}$

In order to evaluate the timing with which artemisinins cause embryotoxicity, El-Dakdoky et al. administered artemether to pregnant rates at three points in gestation: i) preimplantation; ii) during organogenesis; and iii) during the fetal period. The study found that embryolethality was only seen during the period of organogenesis. ${ }^{21}$

To further investigate the reproductive effects of artemisinins on the reproductive tract, Ejiofor et al. administered artemether to ovulating and pregnant rats. The study found that artemether administration during ovulation did not affect the rate of conception, litter size, birth weight or growth rate of pups. In addition, the study found no agonist effect of artemether on uterine smooth muscle. However, artemether did prevent oxytocininduced uterine smooth muscle contraction. This finding may indicate that artemether given around the time of delivery could potentially affect the progress of labor by inhibiting the oxytocin response. However, these findings would need to be confirmed in humans. ${ }^{20}$ The embryolethality and teratogenicity of artemisinins seen in animal studies do not demonstrate any maternal toxic effects.

A further question in exploring the toxic effects of artemisinins in pregnancy is whether all artemisinins cause the same effects through a common denominator, or whether some artemisinins may not have the toxic effects seen in others. In 2008, Clark $e t$ al. published a study that compared the results of administration of artesunate, artemether arteether and dihydroartemisinin in pregnant rats. This study again demonstrated the pattern of embryotoxicity, as well as cardiovascular and skeletal anomalies. Moreover, all four types of artemisinin caused the same pattern of teratogenicity, indicating that dihy- droartemisinin, the common derivative of all artemisinins, is the causal toxicant. ${ }^{25}$ Furthermore, an in vitro study by D'Alessandro et al. suggested that a newer artemisinin derivative, artemisone, might be less antiangiogenic than those artemisinins whose main active metabolite is dihydroartemisinin. $^{38}$

Because the animal studies noted here all show the same pattern of teratogenicity and fetal loss, with a possible mechanism of toxicity established, this creates cause for concern in humans as to whether artemisinins are safe in pregnancy. However, the mechanism of teratogenicity by which artemisinins cause congenital malformations in rats may differ significantly in humans, and therefore the animal data may not be predictive of potential for risk to human fetuses in first trimester. The embryolethality seen with artemisinins is caused by an interruption in erythropoiesis, which in rats occurs within one day, and therefore the risk of interference with the process is high even with single or short-course exposures. Although data is limited in humans, primates require 12 days of exposure to reach the same level of inhibition of erythropoiesis or angiogenesis. Therefore, a three-day course of treatment with artemisinins, as is currently prescribed in both children and adults, would be unlikely to result in pregnancy loss or congenital abnormality. ${ }^{39}$

\section{Inadvertent first trimester exposures}

McGready et al. followed women who received artemisinin derivatives for a variety of reasons, such as prior treatment failure and parasitemia. Among these women, 16 inadvertently received artemisinins in first trimester. Among those 16 pregnancies, 12 resulted in normal live births, 1 was lost to follow up, and 3 had spontaneous abortions at 3.5, 5.5 and 7 weeks after exposure. There was no comparison group, but the overall rate of spontaneous abortion among first-trimester exposures with follow-up data available was $20 \%(3 / 15) .^{34}$

Deen et al. followed up a subcohort of pregnant women who took either artesunate plus sulfadoxine-pyrimethamine or placebo as part of a double-blinded, village-randomized malaria transmission-reduction trial. Women who suspected they were pregnant were encouraged not to participate, but 459 women participated who were pregnant did not realize the pregnancy at the time of the study, or decided to participate despite pregnancy. In the secondary analysis, pregnancy outcomes of women who took placebo and women who chose not to participate were combined. This resulted in 287 women who had exposure to artesunate plus sulfadoxine-pyrimethamine and 172 women who did not. Among the participants for whom data was available, there was no difference in rates of pregnancy loss or con- 
genital malformation, including in women who were exposed in first trimester. However, this study is limited by selection bias. Furthermore, the rate of pregnancy loss in first trimester may be under-reported because the pregnancies were not known at the time of drug administration, and this data was only collected later. ${ }^{27}$ A 2001 study by McGready et al. followed 461 women who received a variety of artemisinin-based treatments for malaria. Among these women, 44 were in first trimester, and 20 of the first-trimester treatments were inadvertent. There was no difference in the rates of spontaneous abortion, stillbirth, congenital anomaly or preterm birth in the 44 women treated with artemisinins in first trimester as compared with the general population of the community in which the study was conducted. ${ }^{31}$

\section{Other human safety studies}

In the 2001 McGready study discussed above, the primary outcome of the study was treatment efficacy and is discussed below, but delivery information was available for 414 of the women. There were two maternal deaths; one was due to severe malaria in a primigravida, and the other was unrelated to malaria. Rates of adverse events were compared with rates seen in the community in data collected over a similar time period (1993-2000). The pregnancy outcomes between women in any trimester exposed to artemisinins did not differ from the community. ${ }^{31}$

A 2001 prospective cohort study followed women who were treated for malaria with either artemether-lumefantrine or sulfadoxine-pyrimethamine. The study found that $6 / 159(3.8 \%)$ of pregnancies exposed to artemether-lumefantrine in the first trimester resulted in spontaneous abortion, whereas none of the 153 pregnancies exposed to sulfadoxine-pyrimethamine in the first trimester resulted in abortion. However, there were some confounding factors within this study. More of the women in the artemether-lumefantrine group had laboratory-confirmed malaria than the sulfadoxine-pyrimethamine group, and malaria itself is a risk factor for spontaneous abortion. Furthermore, the women in the artemether-lumefantrine group who had spontaneous abortions had other risk factors, such as repeated episodes of malaria (up to 3), concomitant syphilis, and exposure to salbutamol for threatened abortion. There was no clear time relationship between artemether exposure and abortion. Notwithstanding the higher rate of spontaneous abortion in the artemether-lumefantrine group, the actual perinatal mortality rate between groups did not differ, because the sulfadoxine-pyrimethamine group had a higher rate of preterm birth. There was no difference in the rate of congenital anomalies between the two groups (6.9\% for artemetherlumefantrine and $6.6 \%$ for sulfadoxinepyrimethamine). ${ }^{22}$

A case series by Adam et al. in 2004 treated 28 pregnant women with malaria who failed treatment with quinine and chloroquine. In this study, outcomes were generally good, although there was no comparison group. One woman delivered prematurely 8 weeks after drug administration, but preterm delivery can have many factors, including malaria infection. Only one woman was in first trimester at the time of treatment; she was 10 weeks pregnant. Her pregnancy resulted in a live birth. The other subjects were in second or third trimester, and the mean gestational age was 27 weeks. $^{18}$

A 2007 randomized clinical trial by Kabonge Kaye et al. compared artemether-lumefantrine with chlorproguanil-dapsone, an antifolate combination regimen with an established record of safety in pregnancy, in treatment of uncomplicated malaria in 110 pregnant women and found similar efficacy and safety profiles, as well as treatment efficacy of $100 \%$ in both arms. ${ }^{15}$

In 2007, Dellicour et al. published a review of available data for artemisinins in pregnancy. The pooled data of 14 studies contained 945 women, of whom 123 were in the first trimester. The review concluded that although artemisinins appear safe and effective in pregnancy, the sample sizes were small and insufficient to detect less common adverse outcomes, or to adequately assess the risks of exposure in first trimester. ${ }^{40}$

\section{Pharmacokinetic studies}

A 2006 pharmacokinetic study by McGready et al. looked at dihydroartemisinin levels in 24 pregnant women with prior treatment failure of other agent who were then treated with 3 days of artesunate plus atovaquone plus proguanil. Cure rates were $100 \%$, but it was found that dihydroartemisinin was rapidly cleared in pregnancy, and plasma levels of dihydroartemisinin are lower than previously published data in non-pregnant adults. The study did not have a cohort of non-pregnant adults, and only compared with previous data. ${ }^{28}$ A similar study in 2006 by McGready et al. of artemether-lumefantrine in quinine-resistant malaria found a similar reduction in plasma levels of both artemether and dihydroartemisinin in 13 pregnant women. Artemether was rapidly metabolized to dihydroartemisinin, which was then rapidly eliminated, although treatment efficacy was $100 \%{ }^{13}$

Onyamboko et al. evaluated the pharmacokinetics and pharmacodynamics of a single dose of artesunate in 26 HIV-negative pregnant women with asymptomatic Plasmodium falciparum parasitemia, and used as comparison groups the same women at 3 months postpar- tum, as well as a control group of 25 non-pregnant adult women. Among the pregnant women, 13 were between 22 and 26 weeks gestational age, and 13 were between 32 and 36 weeks gestational age. In all participants, parasite clearance was very high. The AUC was lower in pregnant participants, but there was no difference seen in the rate of parasite clearance despite the lower AUC. Interestingly, when the women were studied at 3 months postpartum, they had the same dihydroartemisinin levels as seen when they were pregnant, and these levels were lower than the non-pregnant control group. ${ }^{29}$ This pharmacokinetic difference indicates that although pregnancy may cause artemisinins to be metabolized more quickly and lead to lower serum concentrations, it is not clear whether there is a difference in clinical effectiveness of artemisinins in pregnancy. Further research is needed to elucidate this and determine whether a higher dose is needed in pregnant women.

\section{Treatment efficacy}

Early studies on artemisinins required enrollment of only subjects who had failed other treatments because artemisinin-based treatment was not yet recommended by the WHO as first line therapy for malaria. An early study in 1998 by McGready et al. administered artemisinins to pregnant women that precluded treatment with non-artemisinins for several reasons. These women either had previously failed quinine or mefloquine, could not tolerate oral medication, or had hyperparasitemia. There was another group of women who received an artemisinin because they were not aware or did not disclose their pregnancy at the time of administration. The inadvertent first-trimester exposures are discussed above. A total of 83 women were treated with an artemisinin derivative, but they did not all receive the same regimen. Treatment failure was defined as reappearance of parasitemia within 42 days of treatment, although genotyping to differentiate recrudescence from reinfection was not done. The majority, 61 women, received artesunate alone, and there were 6 treatment failures, or $9.8 \%$. Twenty-six women received artesunate plus mefloquine, and there were 3 treatment failures, or $11.5 \%$. Three women received artesunate plus mefloquine and there was one treatment failure, but this number is very small and difficult to draw conclusions from. Although treatment failures did occur with the various artemisinin derivatives, the rate of treatment failure was substantially less than the failure rates for quinine and mefloquine in that area, which were $37 \%$ and $36 \%$ respectively. Use of the artesunate as a single agent may have contributed to a higher than expected failure rate, and its combination with mefloquine may not have 
contributed additional effectiveness in such a high resistance setting. ${ }^{34}$

A 2001 study by McGready et al. followed 461 women given a variety of artemisinin-based treatments for malaria. The safety data from this study is discussed above. Because the data collection was done over an 8-year period, the artemisinin regimens vary somewhat, and could not be evaluated individually. The artemisinin given was either artesunate or artemether, and they were given either as single agents, or together, or in combination with mefloquine, atovaquone-proguanil, or clindamycin. The cumulative failure rate by survival analysis for primary treatment was $6.6 \%$ and for recrudescent malaria was $21.7 \%$. No genotyping was done to differentiate recrudescence from new infection. ${ }^{31}$

Another 2001 study by McGready et al. randomized 129 pregnant women with uncomplicated $P$. falciparum or mixed (P. falciparum or $P$. vivax) malaria to either artesunate, or quinine-clindamycin. The treatment efficacy for both arms was $100 \%$ at 42 days post-treatment. Two of the women in the quinine-clindamycin group had reappearance of parasitemia by 42 days, but both were confirmed as new infections by PCR genotyping. ${ }^{30}$

A 2001 prospective study by Bounyasong treated 60 parasitemic women in the second or third trimester of pregnancy with either quinine or artemether. Both groups had 100\% treatment efficacy, but the artemether group had a shorter time to parasite clearance and to fever clearance than the quinine group. ${ }^{33}$

A 2008 retrospective analysis reviewed all cases of 50 pregnant women who were of gestational ages between 9 and 39 weeks who received dihydroartemisinin-piperaquine as treatment for recrudescent malaria. The 63day PCR-adjusted treatment efficacy rate was $92.2 \%{ }^{37}$

In a randomized controlled trial by McGready et al. comparing artemether-lumefantrine with artesunate for both primary and recrudescent malaria, both drugs showed similar cure rates (87-89\%) for primary infection, but artesunate was superior to artemetherlumefantrine in treating recrudescent malaria (Cure rates $91.1 \%$ vs $73.9 \%$, respectively. $\mathrm{P}=0.034){ }^{16}$

A non-inferiority randomized trial in 2010 compared artemether-lumefantrine with quinine in pregnant women. The artemetherlumefantrine proved non-inferior, with a PCRadjusted treatment efficacy of $99.3 \%$ as compared with the efficacy of quinine of $97.6 \%$. However, this study did show reduced levels of lumefantrine on day 7 and this was significantly associated with recurrent parasitemia. ${ }^{17}$

\section{Resistance}

The emergence of artemisinin resistance has been suspected since 2004 and was con- firmed in a 2009 study. The resistance was noted in Southeast Asia, where chloroquine resistance and sulfadoxine-pyrimethamine resistance were also first noted. No resistance has been reported to date in Africa, but with other antimalarials, the emergence of resistance in Africa was delayed relative to Asia and Latin America, but once it reached the continent, it spread rapidly throughout. While still geographically limited, the identification of resistance to artemisinins is concerning because there is currently no drug on the horizon that is sufficiently developed to replace ACTs. ${ }^{10}$

While there is no data currently on artemisinin resistance in pregnancy, pregnant women are of special concern in the emergence of resistance. Pregnant women are more susceptible to malaria and therefore the reduced effectiveness of ACTs would affect them disproportionately. Approval of new medications for pregnant women tends to lag behind approval for non-pregnant adults for justifiable safety reasons. However, with no new medications currently available to replace ACTs, the use of any newly developed medications will have to be further delayed for use in pregnant women. Additionally, pregnant women may, in fact, contribute to patterns of resistance. Pregnant women are not only more susceptible to malaria, but also have higher parasite burdens and more rapid clearance of artemisinins combined with a mildly suppressed immune system. ${ }^{41}$ Therefore, although resistance is typically studied in non-pregnant populations, pregnant women should be considered an important piece of the resistance puzzle.

\section{Co-Infection with HIV}

HIV-infected women have higher susceptibility to malaria and, when infected with malaria, experience higher parasite densities and more severe clinical consequences for mother and fetus. In addition HIV infection eliminates the protective effect of multigravidity, leaving women of HIV-infected pregnant women of all gravidities equally susceptible to malaria. ${ }^{6,42}$ Pregnancy alone compromises a woman's ability to clear malaria infection, and the addition of HIV may compound that effect. ${ }^{43-45}$

Only three of the studies examined in this paper reported the HIV status of the participants. In one study, all participants were HIVuninfected. Both studies that did look at HIV status found that about one-quarter of the participants had HIV, and one of the studies were only able to test $63 \%$ of participants. ${ }^{22,29,32}$ There are no studies evaluating the effectiveness of artemisinins on HIV-infected pregnant women. More research is needed to determine effectiveness of these agents in immunocompromised women.
Because most of the studies did not report the HIV status of the subjects, it is impossible to know whether HIV may have affected the effectiveness of artemisinins, and whether that may account for some variation in the treatment efficacy rates seen among different studies. Future trials of artemisinins in pregnancy should report HIV status of subjects.

\section{Discussion}

There is a growing body of evidence in favor of the use of artemisinins in pregnancy; however there are a number of unanswered questions that would benefit from further research. Artemisinins do appear to be safe in second and third trimester, but data from animal studies indicates that there is a teratogenic and embryolethal effect in rats, guinea pigs and rabbits. It is not clear whether this effect is reflected in humans, and there are some significant differences in human organogenesis as compared with rodent organogenesis that could have a protective effect for human fetuses. The human studies conducted to date do not provide enough evidence to conclusively identify or negate an embryotoxic effect of artemisinins in the first trimester of pregnancy. It is clear, however, that artemisinins are highly effective in pregnancy. Despite pharmacokinetic studies demonstrating lower plasma concentrations of artemisinins in pregnant women as compared with non-pregnant adults, most studies do not indicate that the lower plasma concentrations lead to decreased efficacy, or require increased dosage. However, the study by Piola et al. did show reduced lumefantrine levels, which has been shown to predict efficacy in non-pregnant patients, and this finding was associated with recurrent parasitemia. $^{17}$

Most of the studies of artemisinins in pregnancy demonstrate high levels of efficacy. However, the two largest studies do demonstrate a higher failure rate than other studies (Table 1). It is suggested by the authors that the reduced efficacy in one of the studies is due to the reduced circulating plasma concentrations. ${ }^{13}$ This study was conducted on the northwest border of Thailand, an area in which resistance patterns are often first identified. It was not a randomized trial, but rather a prospective observational study, and therefore the artemisinin treatments were not standardized. While none of the randomized trials showed reduced effectiveness to this degree, the observational studies, which generally had larger sample sizes, did. More research is needed to clarify why this study had reduced effectiveness while the others did not.

It has been suggested that a longer treatment course may be warranted in pregnancy, 
but this prediction was made using modeling, and in vivo studies would be required to demonstrate the effectiveness of this regimen before it could be recommended. ${ }^{46,47}$

Artemisinins appear to be most effective in pregnancy when combined with another antimalarial agent. The short half-life of artemisinins leave patients vulnerable to novel infection soon after treatment, and so combining the artemisinin with a long-acting antimalarial provides optimal treatment. In addition, combination therapy reduces the potential for resistance against artemisinins. Lastly, additional research is needed to investigate specifically vulnerable populations, such as HIV-infected pregnant women, and uses of artemisinins other than treatment, such as intermittent preventive treatment in pregnancy. Malaria in any trimester of pregnancy has serious deleterious effects on the mother and the fetus. Artemisinins are a powerful tool to combat malaria, especially multidrug-resistant parasites. With the WHO recommendations in favor of use of artemisinins in second and third trimester of pregnancy, pregnant women have access to the most potent antimalarial drugs. However, further exploration of the safety of artemisinins in first trimester is warranted in order to definitively accept or reject the use of artemisinins in first trimester. This issue is not insignificant, as malaria is a major cause of early pregnancy loss, and has the potential to interfere with placental implantation and growth, setting the stage for subsequent low birth weight, preterm birth, stillbirth and neonatal demise.

\section{References}

1. Guyatt HL, Snow RW. The epidemiology and burden of Plasmodium falciparumrelated anemia among pregnant women in sub-Saharan Africa. Am J Trop Med Hyg 2001;64:36-44.

2. Desai M, Terkuile F, Nosten F, et al. Epidemiology and burden of malaria in pregnancy. Lancet Infect Dis 2007;7:93104.

3. Rogerson SJ, Hviid L, Duffy PE, et al. Malaria in pregnancy: pathogenesis and immunity. Lancet Infect Dis 2007;7:105-17.

4. Beeson JG, Duffy PE. The immunology and pathogenesis of malaria during pregnancy. Curr Top Microbiol Immunol 2005;297:187227.

5. Whitty C, Edmonds S, Mutabingwa T. Malaria in pregnancy BJOG. 2005;112: 1189-95.

6. ter Kuile F, Parise M, Verhoeff F. The burden of co-infection with human immunodeficiency virus type 1 and malaria in pregnant women in sub-Saharan Africa. Am J
Trop Med Hyg 2004;71:41-54.

7. Guyatt HL, Snow RW. The epidemiology and burden of Plasmodium falciparumrelated anemia among pregnant women in sub-Saharan Africa. Am J Trop Med Hyg 2001;64:36-44.

8. Greenwood B, Alonso P, Kuile ter FO, et al. Malaria in pregnancy: priorities for research. Lancet Infect Dis 2007;7:169-74.

9. Cui L, Su X. Discovery, mechanisms of action and combination therapy of artemisinin. Expert Rev Anti Infect Ther 2009;7:999-1013.

10. Dondorp AM, Yeung S, White L, et al. Artemisinin resistance: current status and scenarios for containment. Nat Rev Microbiol 2010;8:272-80.

11. World Health Organization. Guidelines for the Treatment of Malaria. 2010. WHO, Geneva. Available from: http://www.who. int/malaria/publications/atoz/ 9789241547 925 /en/index.html/

12. Rosenthal PJ. Artesunate for the treatment of severe falciparum malaria. NEJM 2008; 358:1829-36.

13. Mcgready R, Stepniewska K, Lindegardh $\mathrm{N}$, et al. The pharmacokinetics of artemether and lumefantrine in pregnant women with uncomplicated falciparum malaria. Eur J Clin Pharmacol 2006;62: 1021-31.

14. Sowunmi A, Oduola AM, Ogundahunsi OA, et al. Randomised trial of artemether versus artemether and mefloquine for the treatment of chloroquine/sufadoxinepyrimethamine-resistant falciparum malaria during pregnancy. J Obstet Gynaecol 1998;18:322-7.

15. Kaye DK, Nshemerirwe R, Mutyaba TS, Ndeezi G. A randomized clinical trial comparing safety, clinical and parasitological response to artemether-lumefantrine and chlorproguanil-dapsone in treatment of uncomplicated malaria in pregnancy in Mulago hospital, Uganda.J Infect Dev Ctries 2007;2:135-9.

16. Mcgready R, Tan S0, Ashley EA, et al. A randomised controlled trial of artemetherlumefantrine versus artesunate for uncomplicated plasmodium falciparum treatment in pregnancy. PLoS Med 2008; 5:e253.

17. Piola P, Nabasumba C, Turyakira E, et al. Efficacy and safety of artemether-lumefantrine compared with quinine in pregnant women with uncomplicated Plasmodium falciparum malaria: an openlabel, randomised, non-inferiority trial. Lancet Infect Dis 2010;10:762-9.

18 Adam I, Elwasila E, Mohammed Ali DA, et al. Artemether in the treatment of falciparum malaria during pregnancy in eastern Sudan. Trans R Soc Trop Med Hyg 2004;98:509-13.
19. Chen LJ, Wang MY, Sun WK, Liu MZ. [Embryotoxicity and teratogenicity studies on artemether in mice, rats and rabbits]. Acta Pharmacol Sin 1984;5:118-22.

20. Ejiofor JI, Kwanashie HO, Anuka JA. Some pregnancy-related effects of artemether in laboratory animals. Pharmacology 2006;77:166-70.

21. El-Dakdoky MH. Evaluation of the developmental toxicity of artemether during different phases of rat pregnancy. Food Chem Toxicol 2009;47:1437-41.

22. Manyando C, Mkandawire R, Puma L, et al. Safety of artemether-lumefantrine in pregnant women with malaria: results of a prospective cohort study in Zambia. Malar J 2010;9:249.

23. Xu JH, Zhang YP. [Contragestational effects of dihydroartemisinin and artesunate]. Acta Pharm Sinica 1996;31:65761.

24. Clark RL, White TEK, A Clode S, et al. Developmental toxicity of artesunate and an artesunate combination in the rat and rabbit. Birth Defects Res B Dev Reprod Toxicol 2004;71:380-94.

25. Clark RL, Lerman SA, Cox EM, et al. Developmental toxicity of artesunate in the rat: comparison to other artemisinins, comparison of embryotoxicity and kinetics by oral and intravenous routes, and relationship to maternal reticulocyte count. Birth Defects Res B Dev Reprod Toxicol 2008;83:397-406.

26. Li Q, Si Y, Xie L, et al. Severe embryolethality of artesunate related to pharmacokinetics following intravenous and intramuscular doses in pregnant rats. Birth Defects Res B Dev Reprod Toxicol 2009;86:385-93.

27. Deen JL, Seidlein von L, Pinder M, et al. The safety of the combination artesunate and pyrimethamine-sulfadoxine given during pregnancy. Trans R Soc Trop Med Hyg 2001;95:424-8.

28. Mcgready R, Stepniewska K, Ward SA, et al. Pharmacokinetics of dihydroartemisinin following oral artesunate treatment of pregnant women with acute uncomplicated falciparum malaria. Eur J Clin Pharmacol 2006;62:367-71.

29. Onyamboko MA, Meshnick SR, Fleckenstein L, et al. Pharmacokinetics and pharmacodynamics of artesunate and dihydroartemisinin following oral treatment in pregnant women with asymptomatic Plasmodium falciparum infections in Kinshasa DRC. Malar J 2011;10:49.

30. Mcgready R, Cho T, Samuel, et al. Randomized comparison of quinine-clindamycin versus artesunate in the treatment of falciparum malaria in pregnancy. Trans R Soc Trop Med Hyg 2001;95:651-6.

31. Mcgready R, Cho T, Keo NK, et al. Artemisinin antimalarials in pregnancy: a 
prospective treatment study of 539 episodes of multidrug-resistant Plasmodium falciparum. Clin Infect Dis 2001;33:2009-16.

32. Kalilani L, Mofolo I, Chaponda M, et al. A randomized controlled pilot trial of azithromycin or artesunate added to sulfadoxine-pyrimethamine as treatment for malaria in pregnant women. PLoS ONE 2007;2:e1166.

33. Bounyasong S. Randomized trial of artesunate and mefloquine in comparison with quinine sulfate to treat $\mathrm{P}$. falciparum malaria pregnant women. J Med Assoc Thai 2001;84:1289-99.

34. Mcgready R, Cho T, Cho JJ, et al. Artemisinin derivatives in the treatment of falciparum malaria in pregnancy. Trans R Soc Trop Med Hyg 1998;92:430-3.

35. Clark RL. Embryotoxicity of the artemisinin antimalarials and potential consequences for use in women in the first trimester. Reprod Toxicol 2009;28: 285-96.

36. Longo M, Zanoncelli S, Manera D, et al. Effects of the antimalarial drug dihydroartemisinin (DHA) on rat embryos in vitro. Reprod Toxicol 2005;21:83-93.
37. Rijken MJ, Mcgready R, Boel ME, et al. Dihydroartemisinin-piperaquine rescue treatment of multidrug-resistant Plasmodium falciparum malaria in pregnancy: a preliminary report. Am J Trop Med Hyg 2008;78:543-45.

38. D'Alessandro S, Gelati M, Basilico N, et al. Differential effects on angiogenesis of two antimalarial compounds, dihydroartemisinin and artemisone: implications for embryotoxicity. Toxicology 2007;241:66-74.

39. Li Q, Weina PJ. Severe embryotoxicity of artemisinin derivatives in experimental animals, but possibly safe in pregnant women. Molecules 2009;15:40-57.

40. Dellicour S, Hall S, Chandramohan D, Greenwood B. The safety of artemisinins during pregnancy: a pressing question. Malar J 2007;6:15.

41. White NJ, Pongtavornpinyo W, Maude RJ, et al. Hyperparasitaemia and low dosing are an important source of anti-malarial drug resistance. Malar J 2009;8:253.

42. van Eijk AM, Ayisi JG, Kuile ter et al. HIV increases the risk of malaria in women of all gravidities in Kisumu, Kenya. AIDS 2003;17:595-603.
43. Uneke C, Ogbonna A. Malaria and HIV coinfection in pregnancy in sub-Saharan Africa: impact of treatment using antimalarial and antiretroviral agents. Trans $\mathbf{R}$ Soc Trop Med Hyg 2008;103:761-7.

44. Steketee RW, Wirima JJ, Bloland PB, et al. Impairment of a pregnant woman's acquired ability to limit Plasmodium falciparum by infection with human immunodeficiency virus type-1. Am J Trop Med Hyg 1996;55:42-9.

45. Ned R, Moore J, Chaisavaneeyakorn S, Udhayakumar V. Modulation of immune responses during HIV-malaria co-infection in pregnancy. Trends Parasitol 2005; 21:284-91.

46. Tarning J, Mcgready R, Lindegardh N, et al. Population pharmacokinetics of lumefantrine in pregnant women treated with artemether-lumefantrine for uncomplicated Plasmodium falciparum malaria. Antimicrob Agents Chemother 2009;53: 3837-46.

47. Gutman J and Kachur SP. Treating malaria in pregnant women: a pressing problem. Lancet Infect Dis 2010;10:739-40. 